\title{
A DYNAMIC NON-LOCAL MEANS ALGORITHM FOR MONOCHROME IMAGE DENOISING
}

\author{
Jianqiang Gao and Srinivas Nowduri ${ }^{\mathrm{b}}$

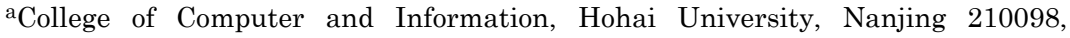 \\ P. R. China \\ ${ }^{\text {b}}$ Department of SS-LET, FSC, 2350 Braodhollow Road, Farmingdale NY 11735, \\ USA
}

\begin{abstract}
The image denoising is the fundamental problem in the field of image processing. The denoising algorithm of image is most commonly designed for removal of both impulsive noise and Gaussian noise. In image denoising process, impulsive noise (salt and pepper noise and random valued impulsive noise) is a most common noise which affects the image quality during image acquisition, transmission, reception or storage. Many classical denoising techniques have evolved over the years, such as mean filter, median filter, and morphological filter. In this paper, a novel dynamic non-local means algorithm has been proposed for monochrome image processing. The experimental results will confirm that proposed method yields the better performance in terms of peak signal to noise ratio (PSNR).
\end{abstract}

Keywords: image denoising, salt and pepper noise, non-local means filter.

\footnotetext{
${ }^{*}$ Corresponding author.

E-mail address: jianqianggaohh@126.com (Jianqiang Gao).
}

Copyright () 2017 Scientific Advances Publishers

2010 Mathematics Subject Classification: 68 U10.

Received April 27, 2017 


\section{Introduction}

Digital image processing technique is widely used in all kinds of fields, such as face recognition [1-10] and biomedical image processing [11-13]. The aim of digital image processing is to improve the potential information for human interpretation and processing of an image data for transmission, storage, and representation for autonomous machine perception. Due to contamination of all kinds of types of noise, such as additive white Gaussian noise, Rayleigh noise, impulsive noise, the image quality degradation. Generally speaking, noise corrupt an image during the processes of acquisition, transmission and reception and storage and retrieval. In real world, in order to obtain good visuality like mobile phone, television, digital cameras, the acquired image signal must be denoised. Image denoising techniques are very important in modern digital image processing. The main goal of image denoising is to reconstruct the original image or the better estimation from the noise data while preserving fine image details. Many denoising algorithms had been proposed, such as information entropy filter [14], optimization of partition-based weighted sum filters [15], nonlocal transform-domain filter [22], based on wavelet filters [16-20], and based on independent component analysis and hierarchical fusion hybrid filter [21]. The performance of image processing can be affected by the presence of salt and pepper noise [22, 23].

Min and Max filters are two classical denoising methods, which can remove salt and pepper noise, respectively. However, the min and max filters can not be work when both types of noise are included in the binary image. Therefore, many researchers proposed all kinds of median filters and morphological operations-based filters. As we all know, median filter is one of the most popular nonlinear filters for removing the impulsive noise, and it has good abilities of keeping edge. But, it requires sorting within the pixel neighbourhood specified by the filter size. The computational burden will increase as the size of the filter increases. In 
addition, some fine details and edges of the image will not be preserved. Due to its simplicity in implementation and also effectiveness in noise suppression, various changes have been done in the filter, such as some modifications median filter (SMF) [24] and center weighted median filter [25]. The conventional median filter applies the median operation to each pixel completely, that is without considering whether it is noisy or noisefee pixel. But still, filtering approaches is subjected and causes for the image degradation. In order to solve the problems of these median filter, switching median filters were introduced in [26, 27]. (i) Impulse detector: a detector analyses local characteristics of the $3 \times 3$ sliding window around each pixel using some considerations and marks the noise pixels in the corresponding local window. (ii) Noise filtering: only the marked pixels are processed rather than the whole area pixels of an image. In [28], a rank-ordered relative differences (RORD-WMF) method to identify corrupted pixels was proposed with sliding window. In [29], a new modified directional weighted median (DWM) filter method was proposed for removal of random-values impulse noise. In [30], the authors proposed a based on lambda multi-diagonal matrix filter method for lowdensity noise removal. Decision tree based denoising (DTBD) method can be found in [31]. The advantage of this method is that it can enhance filtering capability in decreasing impulse noise, while preserving image details.

The remainder of this paper is organized as follows. Section 2 describes the proposed approach in detail. Section 3 reports the experimental results. Section 4 concludes this paper.

\section{The Description of Proposed Denoising Method}

\subsection{Proposed denoising method}

As we all know, Gaussian noise is equally distributed over the signal. Impulsive noise is denoted by changing part of an image pixel with noisy values, not the whole area of an image. Generally, impulsive noise can be 
classified as fixed valued impulse noise and variable type impulse noise. In general, impulse noise can be described by the following two equations. The first equation is strictly following the additive noise model, where the corrupted image $D(x, y)$ is defined as:

$$
D(x, y)= \begin{cases}C(x, y), & 1-P, \\ C(x, y)+N(x, y), & P .\end{cases}
$$

In this equation, $C(x, y)$ is the clean and noise-free image, $N(x, y)$ is the noise intensity value, or the noise amplitude, $P($ i.e., $0 \leq P \leq 1$ ) presents the noise density. Higher value of $P$ means higher corruption level.

Another general impulse noise model uses a simplified version of Equation (1). For this general model, the damaged image $D(x, y)$ is defined as:

$$
D(x, y)= \begin{cases}C(x, y), & 1-P, \\ N(x, y), & P .\end{cases}
$$

This simplification is carried out because for the case of corrupted pixels, the results of $C(x, y)+N(x, y)$ in Equation (1) can actually take any values. This is due to $N(x, y)$ is a random value. Equation (2) shows that the corrupted pixels are directly been replaced with the noise intensity values.

Hence, it is very difficult to remove variable type impulse noise rather than fixed value impulse noise. Our main aim is to preserve the details and restrain the noise of image. The difference between fixed value and variable type impulse noise is shown in Figure 1.

In the case of fixed value impulse noise, the pixel is replaced with noise may be either $N_{\min }(0)$ or $N_{\max }(255)$, where as in variable type impulse noise situation it may range from $N_{\min }$ to $N_{\max }$. 
(a)

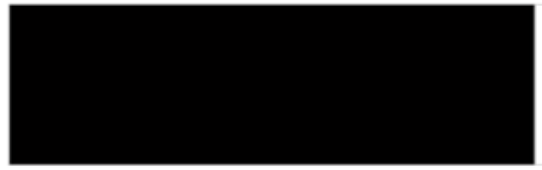

0

(b)
255

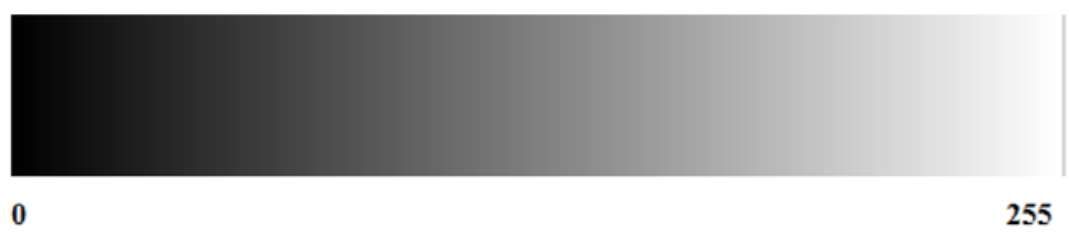

Figure 1. Representation of impulse noise: (a) fixed value impulse noise and (b) variable type impulse noise.

\subsection{Noise detection}

In fact, the level of impulse noise is different for an image. Some pixels look more like corrupted pixels, and some do not look like corrupted pixels. From the theoretical aspect, the independent decision rules should be used for separate levels. So, all pixels should be grouped based on the level of how impulsive-like before making a decision, and adopt different decision rules with different group. In statistics, there are various methods for detecting outliers, such as the ratio between its distance to the sample mean and standard deviation.

In the section, a new detection mechanism has been proposed based on the dynamic statistics. At present, many existing detection methods are often realized by comparing certain number of local neighbourhood statistics to the same number of corresponding thresholds. However, all these existing detection methods without considering the outlying differences among the pixels. The proposed method divides the pixels into four clusters based on the error values. The absolute deviation to the median is used to identify the impulsive noise in each cluster. The good results can be obtain by detecting every pixel, however, the efficiency may be low. Hence, in the new method, all the noisy pixels can be 
detected in the block. The proposed impulse detector should be able to detect most of the corrupted pixels as possible. If there are too many undetected noisy pixels, these pixels will lead to the presence of noticeable noise patches. The results show that our proposed method is more robust to the noise ratio than the other methods.

Non-local means filtering (NLM) estimates an uncorrupted intensity as average weighted for all pixel in the image, and the weighted value are proportional to the similarity between the neighbour pixel value being processed and the surrounding pixels of neighbour pixels. However, these NLM filtering method can preserve image details during denoising. The best solution could be to locally varying parameters, so that they are primely tuned to remove the particular amount and various noises present in each part of the image.

Table 1. The comparison of PSNR (dB) using existing and proposed methods

\begin{tabular}{clccccc}
\hline $\begin{array}{c}\text { Noise } \\
\text { density }\end{array}$ & Test image & SMF [24] & PSMF [27] & AMF [32] & TVIF [33] & Proposed \\
\hline \multirow{2}{*}{$50 \%$} & Lena & 14.99 & 23.08 & 28.41 & 29.12 & $\mathbf{3 0 . 1 8}$ \\
& Cameraman & 14.35 & 17.54 & 25.83 & 26.67 & $\mathbf{2 7 . 0 1}$ \\
& Peppers & 14.84 & 22.85 & 27.49 & 28.57 & $\mathbf{2 8 . 6 1}$ \\
& House & 15.24 & 18.71 & 28.66 & 30.93 & $\mathbf{3 1 . 2 2}$ \\
& Lena & 6.69 & 10.17 & 19.42 & 22.37 & $\mathbf{2 3 . 9 1}$ \\
& Cameraman & 6.42 & 9.49 & 17.41 & 20.18 & $\mathbf{2 0 . 8 2}$ \\
& Peppers & 6.55 & 10.33 & 18.58 & 21.94 & $\mathbf{2 2 . 2 3}$ \\
& House & 6.65 & 10.24 & 18.08 & 23.36 & $\mathbf{2 3 . 6 4}$ \\
\hline
\end{tabular}




\section{Experimental Results}

To make objective assessment, the well-known peak signal to noise ratio (PSNR) is taken as a metric. The PSNR is defined as follows:

$$
P S N R=10 \log _{10}\left(\frac{255^{2}}{(1 / m n) \sum_{i=1}^{m} \sum_{j=1}^{n}[Z(i, j)-A(i, j)]^{2}}\right),
$$

where $Z(i, j)$ and $A(i, j)$ denote the de-noised image and original image, respectively. As we all know, the bigger the PSNR, the better the visual quality of the restored image.

In this section, several experiments were designed to demonstrate the effectiveness of our proposed approach. All these images were added salt and pepper noises with different noise densities, such as 50\% and $90 \%$. The experimental results show that our algorithm not only efficiently removes the salt and pepper noises, but also preserves image details well. In order to show the performance in an all-round way, we compare the proposed approach with SMF [24], PSMF [27], AMF [32], and TVIF [33]. All the experiments are carried out on a PC MATLAB $2010 \mathrm{~b}$ with 2.40GHZ CPU, 3GB RAM. The proposed method and other methods were evaluated according to PSNR. Four standard test images sized $256 \times 256$ as shown in Figure 2 . 


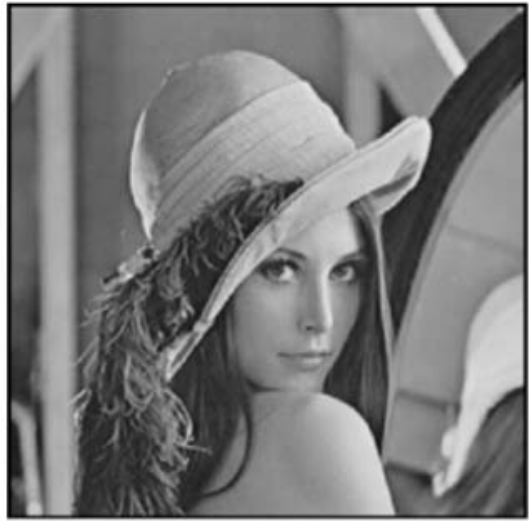

(a) Lena

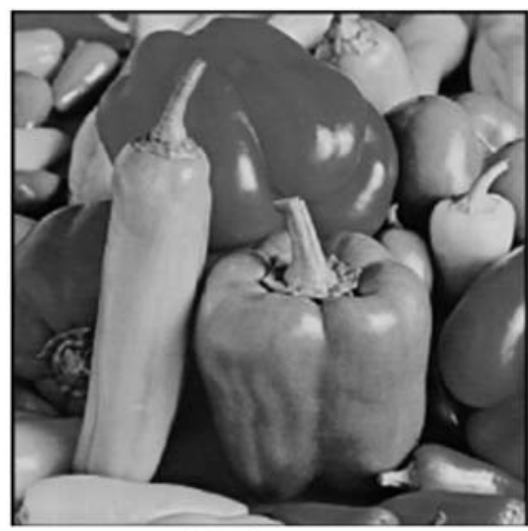

(c) Peppers

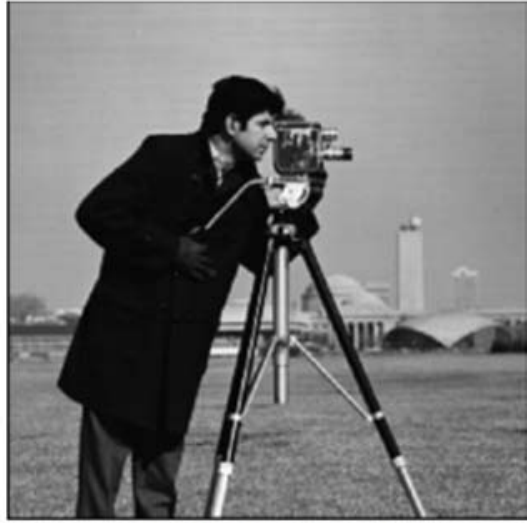

(b) Cameraman

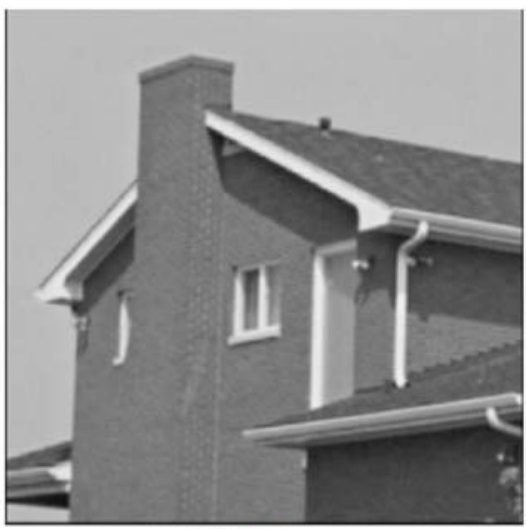

(d) House

Figure 2. Four standard test images.

The corrupted versions of Lena under different noise densities as shown in Figure 3. The PSNR comparisons with respect to four standard gray images among different methods are list in Table 1, where the best results were marked bold. From Table 1, we can clearly to see that the PSNR values of our proposed method are all bigger than those of other compared algorithms. This means that our method is better than other methods in visual quality. Figures 4-5 present visual quality comparisons among different methods under different corrupted versions of Lena. 


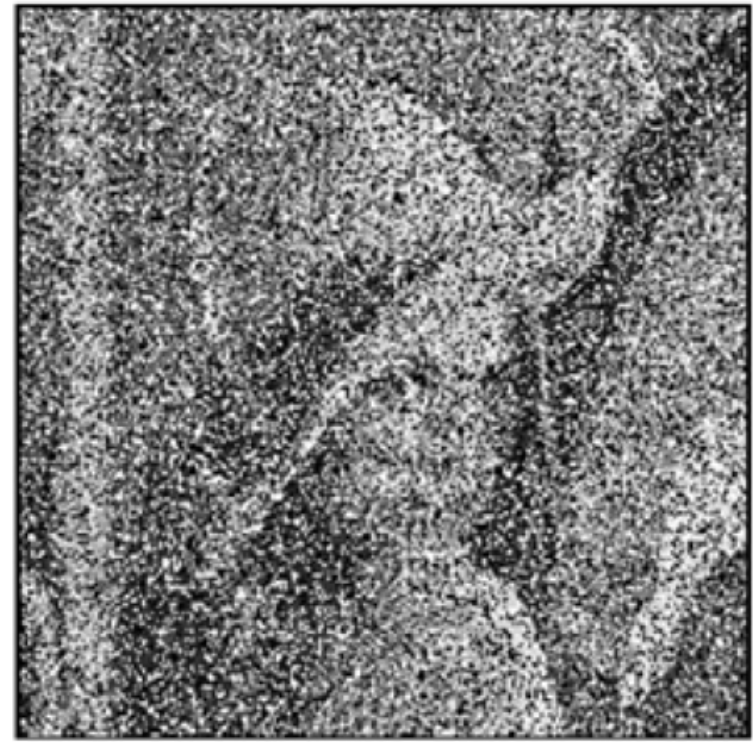

(a) Noise density $50 \%$

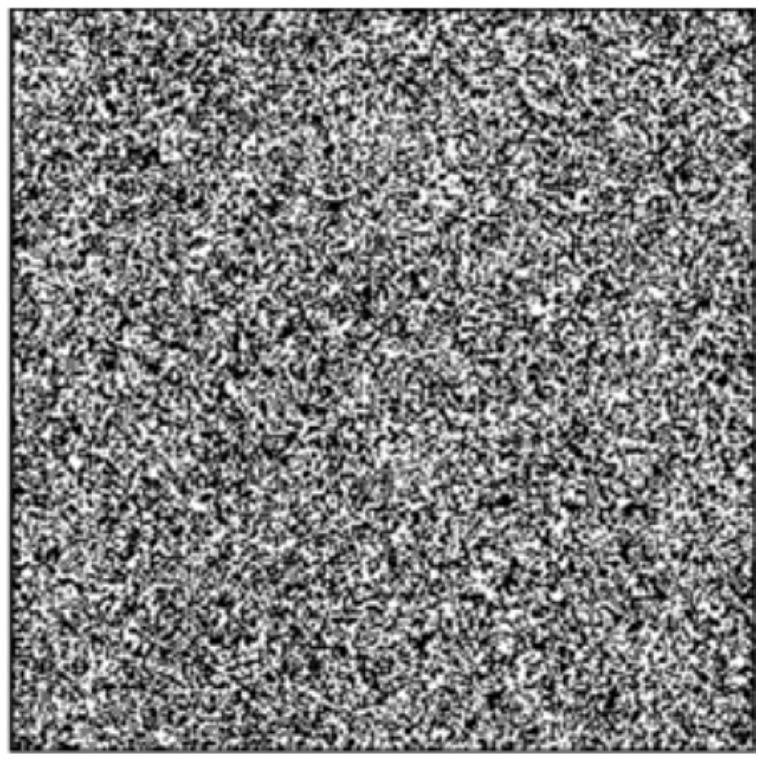

(b) Noise density $90 \%$

Figure 3. The corrupted versions of Lena under different noise densities. 


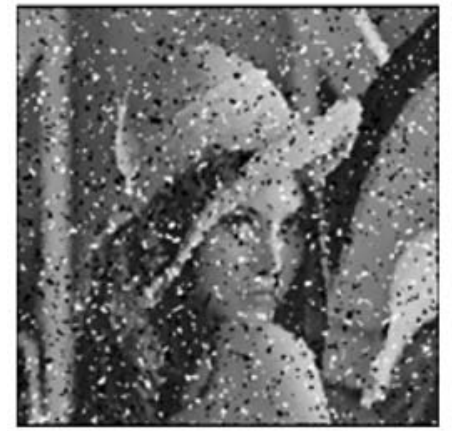

(a)SMF[24]

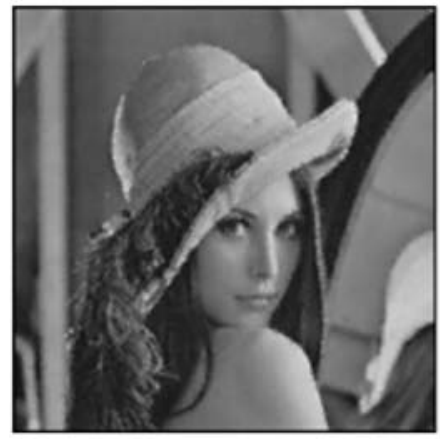

(c)AMF[32]

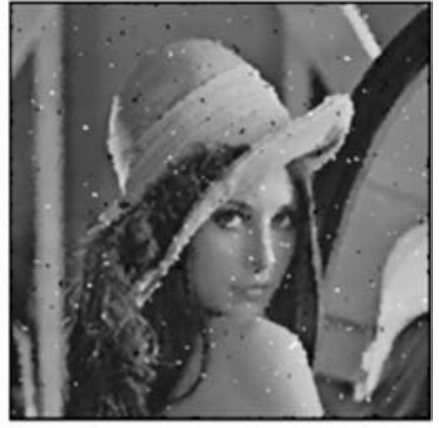

(b)PSMF[27]

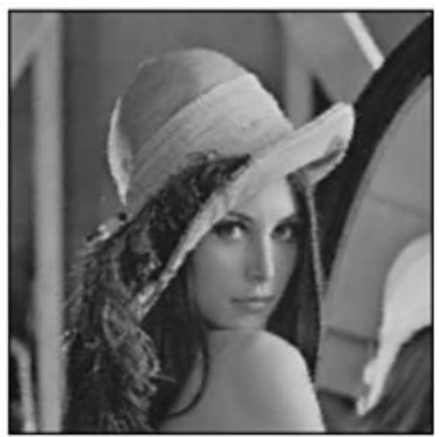

(d)TVIF[33]

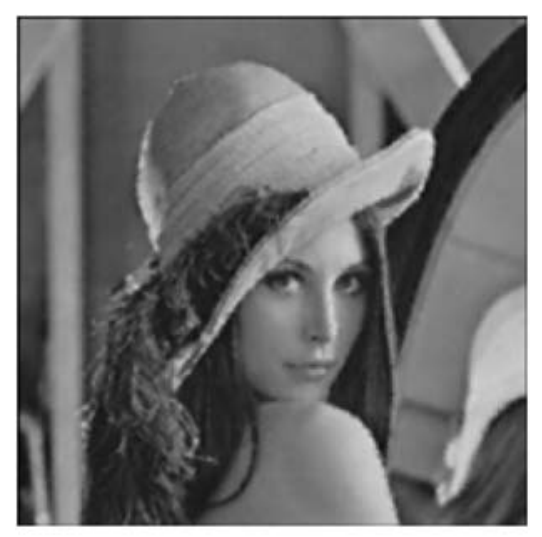

(e)Proposed

Figure 4. Visual quality comparisons among different methods with noise density is $50 \%$. 


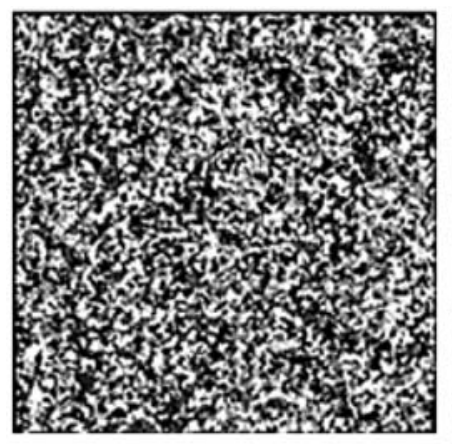

(a)SMF[24]

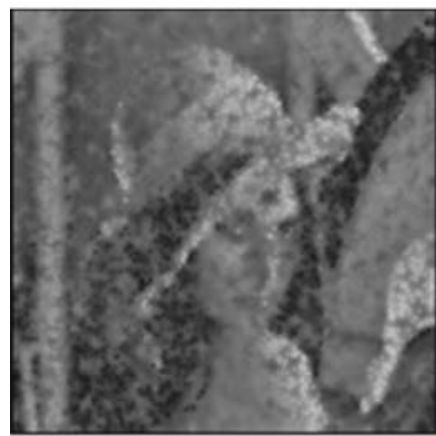

(c)AMF [32]

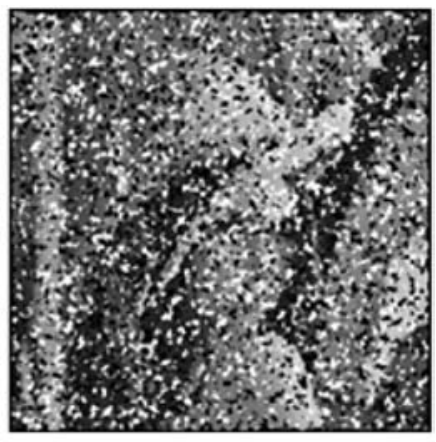

(b)PSMF[27]

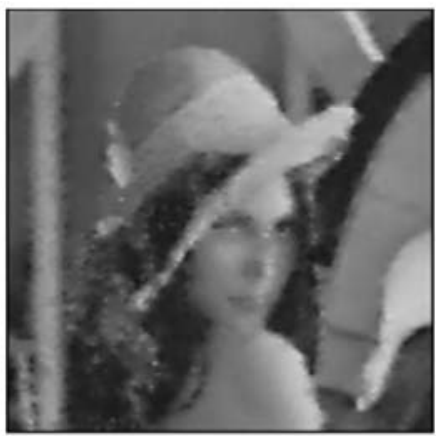

(d)TVIF[33]

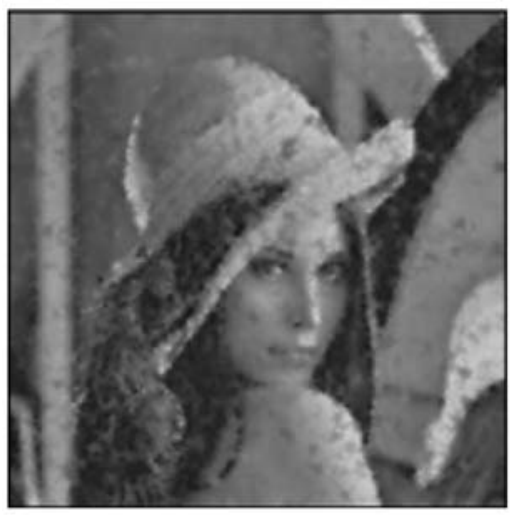

(e)Proposed

Figure 5. Visual quality comparisons among different methods with noise density is $90 \%$. 
From all the above results, the proposed method has better performance in visual quality than the compared methods. This is attributed to our elaborate noise detection. This strategies ensure that our method can efficiently remove noise while preserving image details even at a high noise density.

\section{Conclusion}

In this paper, an efficient dynamic non-local means method was proposed for removing salt and pepper noise. The proposed method divides the pixels into four clusters based on the error values. And the absolute deviation to the median is used to identify the impulsive noise in each cluster. Hence, in the new method, all the noisy pixels can be detected in the block. The proposed impulse detector should be able to detect most of the corrupted pixels as possible. The results have shown that proposed method can efficiently remove salt and pepper noise from corrupted images, and outperforms some well-known algorithms.

Competing Interests. The authors declare that they have no competing interests.

\section{References}

[1] Q. Gao, L. Zhang and D. Zhang, Face recognition using FLDA with single training image per person, Applied Mathematics and Computation 205(2) (2008), 726-734.

[2] M. Koc and A. Barkana, A new solution to one sample problem in face recognition using FLDA, Applied Mathematics and Computation 217(24) (2011), 10368-10376.

[3] J. Gao and L. Fan, Kernel-based weighted discriminant analysis with QR decomposition and its application face recognition, WSEAS Transactions on Mathematics 10(10) (2011), 358-367.

[4] X. Li, S. Fei and T. Zhang, Median MSD-based method for face recognition, Neurocomputing 72(16) (2009), 3930-3934.

[5] J. Gao, L. Fan and L. Xu, Solving the face recognition problem using QR factorization, WSEAS Transactions on Mathematics 11(1) (2012), 728-737.

[6] X. Li, S. Fei and T. Zhang, Weighted maximum scatter difference based feature extraction and its application to face recognition, Machine Vision and Applications 22(3) (2011), 591-595. 
[7] J. Gao, L. Fan and L. Xu, Median null (Sw)-based method for face feature recognition, Applied Mathematics and Computation 219(12) (2013), 6410-6419.

[8] J. Gao, L. Fan, L. Li et al., A practical application of kernel-based fuzzy discriminant analysis, International Journal of Applied Mathematics and Computer Science 23(4) (2013), 887-903.

[9] L. Li, J. Gao and H. Ge, A new face recognition method via semi-discrete decomposition for one sample problem, Optik-International Journal for Light and Electron Optics 127(19) (2016), 7408-7417.

[10] L. Li H. Ge and J. Gao, Maximum-minimum-median average MSD-based approach for face recognition, AEU-International Journal of Electronics and Communications 70(7) (2016), 920-927.

[11] J. E. Iglesias and M R. Sabuncu, Multi-atlas segmentation of biomedical images: A survey, Medical Image Analysis 24(1) (2015), 205-219.

[12] S. Nirmala and V. Palanisamy, Clinical decision support system for early prediction of Down syndrome fetus using sonogram images, Signal, Image and Video Processing $5(2)(2011), 245-255$.

[13] M. Sprindzuk, A. Dmitruk, V. Kovalev et al., Computer-aided image processing of angiogenic histological samples in ovarian cancer, Journal of Clinical Medicine Research 1(5) (2011), 249-261.

[14] J. Zuo, C. Zhao, Q. Pan et al., A novel binary image filtering algorithm based on information entropy, Intelligent Control and Automation, 2006.WCICA 2006. The Sixth World Congress on IEEE 2 (2006), 10375-10379.

[15] M. Shao and K. E. Barner, Optimization of partition-based weighted sum filters and their application to image denoising, Image Processing, IEEE Transactions on 15(7) (2006), 1900-1915.

[16] M. Maggioni, V. Katkovnik, K. Egiazarian et al., Nonlocal transform-domain filter for volumetric data denoising and reconstruction, Image Processing, IEEE Transactions on 22(1) (2013), 119-133.

[17] W. Zhang, F. Yu and H. Guo, Improved adaptive wavelet threshold for image denoising, Control and Decision Conference, CCDC'09. Chinese IEEE (2009), 5958-5963.

[18] Z. J. Xie, B. Y. Song, Y. Zhang et al., Application of an improved wavelet threshold denoising method for vibration signal processing, Advanced Materials Research 889 (2014), 799-806.

[19] R. Yan, L. Shao and Y. Liu, Nonlocal hierarchical dictionary learning using wavelets for image denoising, Image Processing, IEEE Transactions on 22(12) (2013), 46894698.

[20] J. J. Zhong, S. N. Fang and C. Y. Linghu, Research on application of wavelet denoising method based on signal to noise ratio in the Bench Test, Applied Mechanics and Materials 457 (2014), 1156-1162. 
[21] G. Salimi-Khorshidi, G. Douaud, C. F. Beckmann et al., Automatic denoising of functional MRI data: Combining independent component analysis and hierarchical fusion of classifiers, Neuroimage 90 (2014), 449-468.

[22] Z. Ibrahim, N. K. Khalid, I. Ibrahim et al., A noise elimination procedure for printed circuit board inspection system, Modeling \& Simulation, 2008. AICMS 08. Second Asia International Conference on IEEE (2008), 332-337.

[23] A. Graf, A. J. Smola and S. Borer, Classification in a normalized feature space using support vector machines, Neural Networks, IEEE Transactions on 14(3) (2003), 597-605.

[24] T. Nodes and N. Gallagher, Median filters: Some modifications and their properties, IEEE Transactions on Acoustics, Speech, and Signal Processing 30(5) (1982), 739-746.

[25] S. J. Ko and Y. H. Lee, Center weighted median filters and their applications to image enhancement, IEEE Transactions on Circuits and Systems 38(9) (1991), 984-993.

[26] T. Sun and Y. Neuvo, Detail-preserving median based filters in image processing, Pattern Recognition Letters 15(4) (1994), 341-347.

[27] Z. Wang and D. Zhang, Progressive switching median filter for the removal of impulse noise from highly corrupted images, IEEE Transactions on Circuits and Systems II: Analog and Digital Signal Processing 46(1) (1999), 78-80.

[28] H. Yu, L. Zhao and H. Wang, An efficient procedure for removing random-valued impulse noise in images, IEEE Signal Processing Letters 15 (2008), 922-925.

[29] Y. Dong and S. Xu, A new directional weighted median filter for removal of randomvalued impulse noise, IEEE Signal Processing Letters 14(3) (2007), 193-196.

[30] L. Li, H. Ge, Y. Zhang and J. Gao, Low-density noise removal based on lambda multi-diagonal matrix filter for binary image, Neural Computing and Applications (2016). doi:10.1007/s00521-016-2538-7

[31] S. Geetha, N. Ishwarya and N. Kamaraj, Evolving decision tree rule based system for audio stego anomalies detection based on Hausdorff distance statistics, Information Sciences 180(13) (2010), 2540-2559.

[32] C. C. Chang, J. Y. Hsiao and C. P. Hsieh, An adaptive median filter for image denoising, Intelligent Information Technology Application, 2008. IITA'08. Second International Symposium on IEEE 2 (2008), 346-350.

[33] J. Wu and C. Tang, An efficient decision-based and edge-preserving method for saltand-pepper noise removal, Pattern Recognition Letters 32(15) (2011), 1974-1981. 